\title{
Carbon-Nanotube Based Thermoelectrical Paste for Enhancing Solar Cell Effciency
}

\author{
Mohamed Fathi Sanad ${ }^{1}$, Ahmed Shaker $^{1}$, Sameh O. Abdellatif ${ }^{1}$, Iman Elmahallawi ${ }^{1}$, Hani A. Ghali ${ }^{1}$, and \\ Khaled $\mathrm{Kirah}^{2}$ \\ ${ }^{1}$ Centre for Emerging Learning Technologies (CELT), The British University in Egypt, Cairo, Egypt \\ ${ }^{2}$ Engineering Physics and Mathematics Department, Ain Shams University, Cairo, Egypt
}

\begin{abstract}
A super-passive cooling technique based on a thermal paste is proposed for PV efficiency enhancement in elevated temperature conditions. A mixture between carbon nanotubes and graphene having a promising Seebeck coefficient is chosen. An overall enhancement in efficiency by around $58 \%$ was reached while thermoelectrically supplying hundreds of micro-Watt per PV Watt.
\end{abstract}

\section{Introduction}

Nowadays, the need for energy is increasing with population and economic growth around the world. The global energy demand is expected to increase to 16 Billion Tons of Oil Equivalent (TOE) in 2030 (1 TOE $\approx$ 42 GJ) [1], which is a huge amount of energy that need to be provided. Most of the energy provided today comes from fossil fuel that releases huge amounts of greenhouse gases which are the primary cause of global warming. In 2013 the energy related greenhouse-gas emissions was 35.5 Gigaton, and according to the Intended Nationally Determined Contributions (INDCs), it will increase to 38.3 Gigaton in 2030 [2]. This shows the need to more green and renewable energy sources.

Thermoelectric generators (TEGs) are relatively new and promising devices that are used to harvest energy from any temperature gradient. These devices are based on the Seebeck effect in which a temperature difference between two dissimilar electrical conductors or semiconductors produces a voltage difference between the two substances. The max efficiency of a thermoelectric device is given by:

$$
\eta_{\max }=\frac{T_{h}-T_{c} \sqrt{1+Z\left(T_{m}\right)}-1}{T_{h} \sqrt{1+Z\left(T_{m}\right)}+\frac{T_{c}}{T_{h}}}
$$

where $T_{h}$ and $T_{c}$ are respectively the hot and the cold side temperatures, $T_{m}=\frac{T_{h}+T_{c}}{2}$ and $Z\left(T_{m}\right)$ is the device figure of merit defined as:

$$
Z\left(T_{m}\right)=\frac{S^{2} T_{m}}{\rho k_{\text {total }}}
$$

where $S$ is the Seebeck coefficient, $\rho$ is the electrical resistivity and $k_{\text {total }}$ is the total thermal conductivity [4].
Therefore, researchers are working on developing new materials with higher Seebeck coefficient.

Semiconductors show superior thermoelectric behavior than metals due to their low thermal conductivity and high Seebeck coefficient. In 2004, Z(T) enhancement using nanostructures was first reported. Lead telluride (PdTe) has been used in TEGs since the 1960s. Nanostructured PdTe shows a higher efficiency $(11 \%$ for $\Delta \mathrm{T}$ of $590 \mathrm{~K})$ over the non-nanostructured form $(5.1 \%$ for a $\Delta \mathrm{T}$ of $417 \mathrm{~K})$ by about $40-50 \%$. This is mainly because of the lower lattice thermal conductivity due to the nanostructuring [5]. Carbon-nanotube has shown impressive thermoelectric behavior. A value of $Z(T)=0.02$ has been reached using carbon nanotube (CNT)-filled polymer composites which is at least one order of magnitude higher that bulk $\mathrm{Si}$ based thermoelectric generators [6]. In addition, graphene/carbon-nanotube polymer based thermoelectric devices showed the highest electrical conductivity compared to Polymer based ternary thermoelectric composites as reported in [7].

One problem with photovoltaic panels is heating as the efficiency of the panel decreases by $0.45 \%$ for each degree rise over $25{ }^{\circ} \mathrm{C}$ [8]. Thermoelectric devices can be used as a cooling solution for photovoltaic panels. This is a two-fold advantage: increasing the efficiency and generating energy in the same time.

This paper reports the preparation and the properties of graphene/carbon-nanotube/polymer based thermoelectric paste and its potential in photovoltaic cooling and low-grade thermoelectric energy harvesting.

\section{Experimental work}

\subsection{Thermoelectric paste preparation}


Thermoelectric paste was produced using the modified Hummers method from pure graphite powder in a mixture with Multi-Walled Carbon Nanotubes (MWCNTs) [9]. In this method, $60 \mathrm{ml}$ of concentrated sulfuric acid and $6 \mathrm{ml}$ of phosphoric acid were stirred for 10 minutes. Then $0.5 \mathrm{gm}$ of graphite was added into the solution under vigorous stirring. Around $3 \mathrm{gm}$ of the potassium permanganate as strong oxidizing agent were then added slowly into the solution. This mixture was stirred for 12 hours until the mixture became green.

The green mixture is then pureed with $2 \mathrm{ml}$ of hydrogen peroxide slowly while cooling in the ice bath for 30 minutes. $20 \mathrm{ml}$ of hydrochloric acid and $60 \mathrm{ml}$ of deionized water were added and centrifuged for 10 minutes. This was followed by decanting the residuals and then rewashing again with deionized water for 15 times until it became clear.

The solution was stirred well in a mixture of $0.6 \mathrm{gm}$ of MWCNTs and 0.6 gm of graphene oxide purchased from Sigma Aldritch in presence of PEDOT in DMSO as a solvent and stirred for $3 \mathrm{hrs}$ until a homogeneous solution was obtained. Finally, the solution was dried at $60{ }^{\circ} \mathrm{C}$ for $1 \mathrm{hr}$. Different samples were prepared by varying the amount of graphite and CNTs in attempt to obtain pastes with the best thermoelectric behavior according to table 1 .

Table 1. Various ratios for Graphite oxide, graphene oxide and CNT in the prepared samples.

\begin{tabular}{|c|ccc|} 
& $\begin{array}{c}\text { Graphite } \\
\text { oxide }\end{array}$ & $\begin{array}{c}\text { Graphene } \\
\text { oxide }\end{array}$ & CNT \\
MS-TE-001-01 & 1 & 1 & 1 \\
MS-TE-002-01 & 2 & 1 & 1 \\
MS-TE-003-01 & 1 & 1 & 2 \\
MS-TE-004-01 & 1 & 2 & 1
\end{tabular}

\subsection{Light source chractraization}

Solar cells must be characterized under standard conditions using solar simulators. In principal, the high uniformity, temporal stability and spectrum matching are the three main parameters that distinguish solar simulators from other low-cost sources. However, commonly used low-cost light sources can still be used for characterizing PV cells, while taking the mismatching factors in consideration. Herein, a commercial H4 12V 100/90 W Halogen lamp (commonly used in most car lighting) is used [10]. Ocean Optics Red Tide USB650 FiberOptic Spectrometer [11] has been used to optically characterize the used light source.

Light is collected through a fiber terminated by a cosine corrector. This is a preconfigured, ready-to-use spectrometer in the $350-1000 \mathrm{~nm}$ range. Its detector has 650 active pixels, or one data point per nanometer. It uses Sony ILX511 linear silicon CCD array detector and has a sensitivity of up to 75 photons/count at $400 \mathrm{~nm}$. The spectrometer reads the intensity as a number of photons/count (i.e. arbitrary units).
The spectrometer readings have been calibrated by using a reference sample to give readings in Watts/unit area. Figure 1 shows the UV-Vis-IR spectra for the used low-cost light source.

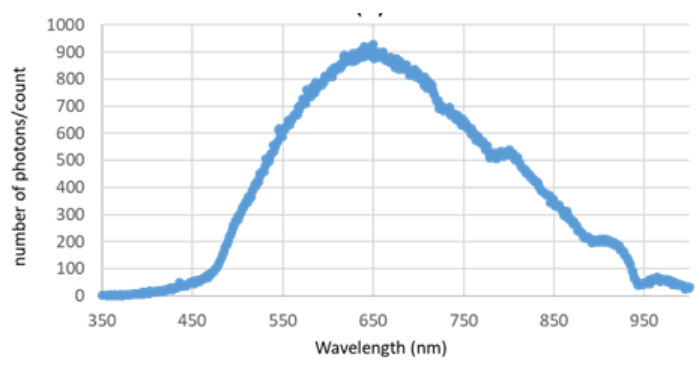

Fig. 1. UV-Vis-NIR spectrum for the selected light source.

The optical mismatching was calculated based on figure 1 with respect to the standard solar irradiance given in AM1.5G [12]. This mismatching factor will be considered in calculating the solar cells efficiency in the next section. Regarding the temporal stability, the selected source showed an acceptable stability with time reaching an overall variation less than $0.1 \%$ across five continuous hours of operation. Finally, to minimize the uniformity mismatching errors, a solar cell under test with small area $(20 \mathrm{~mm} \times 20 \mathrm{~mm})$ is selected where the used light source is assumed to be uniform within this area.

\section{Results and discussion}

\subsection{Thermoelectric paste optimization and chractraization}

Various thermoelectric paste prepared in section 2B are characterized seeking for an optimum recipe. Current, voltage and resistance were measured with varying temperature difference for $\Delta T=0$ to $14{ }^{\circ} \mathrm{C}$ to investigate the optimum thermoelectric behavior among all prepared samples. This temperature range is chosen to cover the expected temperature difference due to solar cell backside where experimental measurements showed a difference of around $10{ }^{\circ} \mathrm{C}$.

Figure 2 presents the behavior of the extracted power with variable temperature difference $(\Delta T)$ between the two sides of the sample. It was observed that sample MSTE-004-01 achieved the highest extracted power of about $82 \mu \mathrm{W}$ at $\Delta T=14{ }^{\circ} \mathrm{C}$. This sample was then used in the backside of the solar cell to examine its utility in enhancing the solar cell efficiency under high temperature.

On the other hand, X-ray powder diffraction (XRD) was performed for the optimum thermoelectric paste. The sample was loaded into a P-Analytical X'PERT MPD diffractometer holder using $(\mathrm{Cu}[\mathrm{K} \alpha 1 / \mathrm{K} \alpha 2])$ rays. Figure 3 shows the obtained pattern. X-ray peaks were observed at a diffraction angle range from $20^{\circ}$ to $60^{\circ}$ with $0.02^{\circ}$ step. A broad peak at $25.9^{\circ}$ corresponding to the $(020)$ 
plane of PEDOT is observed. However, the intensity of the reduced graphene oxide ( $\mathrm{rGO}$ ) and $\mathrm{CNT}$ were very weak due to very low concentrations. Interaction of aggregated $\mathrm{rGO}$, CNTs with PEDOT chains degrade the crystalline phase and the stack structure of PEDOT. On the other hand, there is a strong and sharp peak of graphene oxide which exhibits a (001) reflection at 9.090 corresponding to a basal spacing of $\mathrm{d}_{001}=0.951 \mathrm{~nm}$.

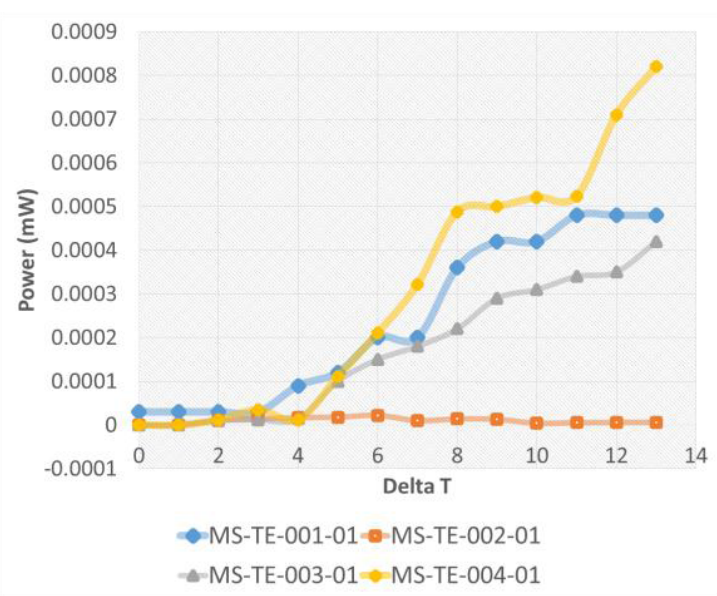

Fig. 2. Extracted power from thermoelectric generators using different CNT recipies.

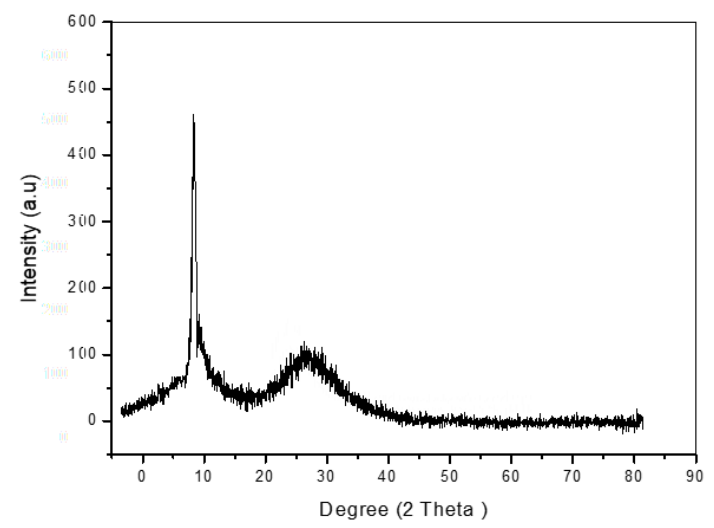

Fig. 3. XRD for the optumum thermoelecteic paste

\subsection{PV module I-V chractaization}

In order to investigate the utility of the thermoelectric paste on the solar cell efficiency, nanocrystalline and amorphous solar cells were used [13]. Figure 4 shows the measured $I-V$ characteristic curves of amorphous silica solar cell with and without the optimized thermoelectric paste at different distances between the cell and the light source. Characterization has been achieved using Keithley 2401 source/meter was used with a four-probe station connection to vary the biased de voltage from $0 \mathrm{~V}$ to $\mathrm{V}_{\mathrm{OC}}$ while acquiring the light generated current.

As observed, varying the distance between the light source and the sample affects both the irradiance level and the temperature as indicated in figure 4. All measurements were made using a Keithley 2401 source/meter.
Figure 4 shows that the thermoelectric paste enhances the performance of the cell regardless of the irradiance level or the temperature. However, this enhancement varies due to the nonlinearity of the thermoelectric behavior shown in figure 2. A maximum enhancement in efficiency reaches $40 \%$ at $55{ }^{\circ} \mathrm{C}$ where the sample was placed $5 \mathrm{~cm}$ from the source. The effect of enhancement is captured from the short circuit current as the open circuit voltage is almost the same.
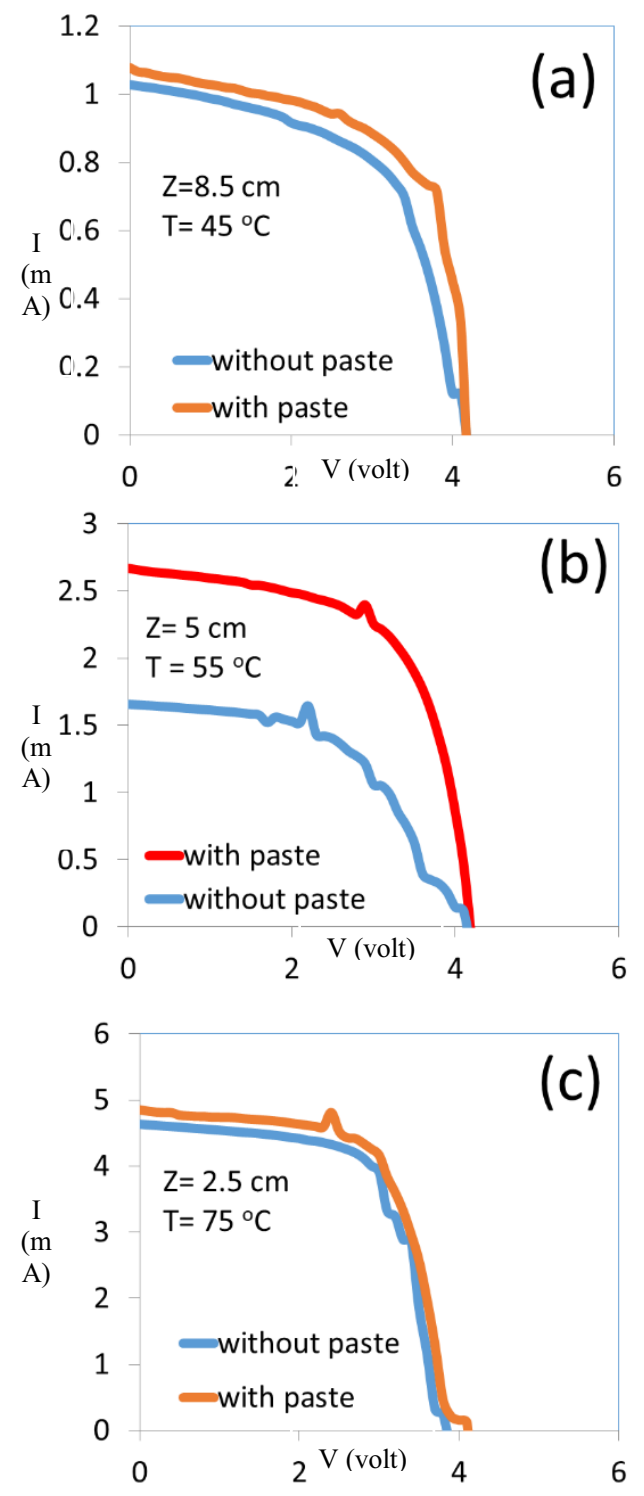

Fig. 4. I-V curve for an amourphous solar cell with and without thermoelectric paste at different distances from light source and different temperature.

The same experiment was repeated on a nanocrystalline solar cell with almost the same dimension as shown in figure 5. Similarly, the use of thermoelectric paste showed better performance on the solar cells where the non-linearity effect can still be detected. The enhancement in efficiency was found to be $58 \%$ at $55{ }^{\circ} \mathrm{C}$ where the sample was placed $5 \mathrm{~cm}$ from the source. 


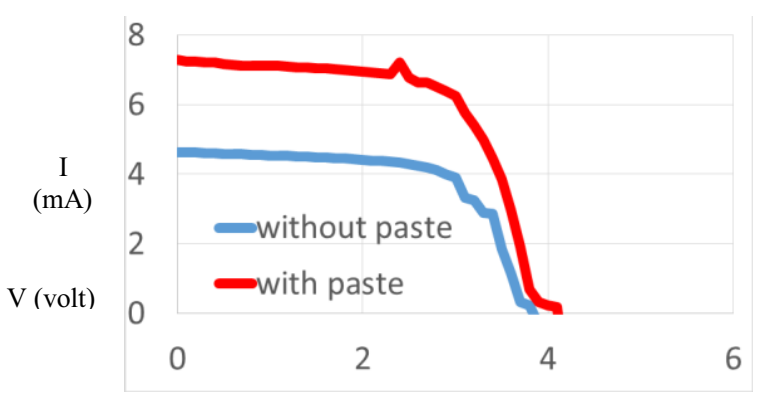

Fig. 5. I-V curves for ananocrystalline solar cell with and without thermoelectric paste for maximum effiency enhancement.

From the presented results in both Fig. 4 and Fig. 5, the utility of using the proposed CNT paste on the backside of solar cells regardless of its type, used irradiance or temperature, is clearly recognized. However, to maximize the efficiency enhancement, optimum theromelectric paste should be used. In addition, the overall performance of nanocrystalline cells is better, with respect to the thermoelectric paste utility, in comparison with amorphous solar cells.

\section{Conclusion}

The work presented in this paper highlighted the potential of utilizing thermoelectric paste for PV cooling especially under high temperatures. A maximum enhancement in efficiency of 58\% was observed for nanocrystalline cells while the backside was pasted with a CNT thermoelectric paste. Amorphous cells also showed an overall enhancement of about $40 \%$ when using the past. Basically, the operating temperature as well as the irradiance level affects the thermal harvesting capabilities, but it is worth to mention that in all the examined cases the thermoelectric paste showed enhanced performance with respect to the reference cell.

\section{Acknowledgment}

The authors would like to acknowledge the support and contribution of the STDF in this work. This work is part of the STDF Project entitled, "Enhancement of Photovoltaic and Wind Turbine Performance for High Temperature and Low Wind Speed Environments", Project ID\#26134.
1. "BP Energy Outlook," BP, 2018.

2. "Energy and Climate Change," INTERNATIONAL ENERGY AGENCY, 2015.

3. G. Jeffrey L, "Carbon-Nanotube-Based Thermoelectric Materials," Advanced materials, p. 35,2018 .

4. H. Jyrki Tervo, "State-of-the-art of Thermoelectric Materials," VTT WORKING PAPERS, p. 29, 2009.

5. A. Y. Xiaokai Hu, "Power generation from nanostructured PbTe-based thermoelectrics: comprehensive development from materials to modules," Royal society of chemistry , p. 13, 2015.

6. C. Y. Dasaroyong Kim, "Improved Thermoelectric Behavior of Nanotube-Filled Polymer Composites with Poly(3,4-ethylenedioxythiophene) Poly(styrenesulfonate)," American Chemical Society, p. 11., 2010.

7. G.XinLi,"Poly(3,4ethylenedioxythiophene)/graphene /carbon nanotube ternary," Elsevier, p. 5, 23 august 2016.

8. C. Cătălin George Popovicia, "Efficiency improvement of photovoltaic panels by using air cooled heat sinks," Elsevier, p. 8, 2015.

9. H. Naeem, M. Ajmal, S. Muntha, j. Ambreen, \&M. Siddiq, "Synthesis and characterization of graphene oxide sheets integrated with gold nanoparticles and their applications to adsorptive removal and catalytic reduction of water contaminants," RSC Advances, 8(7), 3599-3610.

10. T. Gorkhover, M. Adolph, D. Rupp, S. Schorb, S. W. Epp, B. Erk, et al., "Nanoplasma dynamics of single large xenon clusters irradiated with superintense $\mathrm{x}$ ray pulses from the linac coherent light source freeelectron laser," Physical review letters, vol. 108, p. 245005, 2012.

11. P. A. Gilbert and W. Haeberli, "Experiments on subtractive color mixing with a spectrophotometer," American Journal of Physics, vol. 75, pp. 313-319, 2007.

12. S. Abdellatif and K. Kirah, "Numerical Modeling and Simulation for a Radial $p-i-n$ Nanowire Photovoltaic Device," Energy Procedia, vol. 36, pp. 488-491, // 2013.

13. https://media.digikey.com/pdf/Data\%20Sheets/Sany o\%20Energy/Amorphous_Br.pdf

\section{References}

